\title{
Misophonia: current perspectives
}

This article was published in the following Dove Press journal:

Neuropsychiatric Disease and Treatment

18 August 2015

Number of times this article has been viewed

\author{
Andrea E Cavanna ${ }^{1-3}$ \\ Stefano Seri ${ }^{3,4}$ \\ 'Department of Neuropsychiatry, \\ Birmingham and Solihull Mental \\ Health NHS Foundation Trust \\ and University of Birmingham, \\ Birmingham, ${ }^{2}$ Sobell Department of \\ Motor Neuroscience and Movement \\ Disorders, Institute of Neurology, \\ University College London, London, \\ ${ }^{3}$ School of Life and Health Sciences, \\ Aston Brain Centre, Wellcome Trust \\ Laboratory for MEG Studies, Aston \\ University, ${ }^{4}$ Children's Epilepsy \\ Surgery Programme, The Birmingham \\ Children's Hospital NHS Foundation \\ Trust, Birmingham, UK
}

Correspondence: Andrea E Cavanna Department of Neuropsychiatry, National Centre for Mental Health, 25 Vincent Drive, Birmingham BI5 2FG, UK Email a.cavanna@ion.ucl.ac.uk
Abstract: Misophonia is characterized by a negative reaction to a sound with a specific pattern and meaning to a given individual. In this paper, we review the clinical features of this relatively common yet underinvestigated condition, with focus on co-occurring neurodevelopmental disorders. Currently available data on the putative pathophysiology of the condition can inform our understanding and guide the diagnostic process and treatment approach. Tinnitus retraining therapy and cognitive behavior therapy have been proposed as the most effective treatment strategies for reducing symptoms; however, current treatment algorithms should be validated in large population studies. At the present stage, competing paradigms see misophonia as a physiological state potentially inducible in any subject, an idiopathic condition (which can present with comorbid psychiatric disorders), or a symptomatic manifestation of an underlying psychiatric disorder. Agreement on the use of standardized diagnostic criteria would be an important step forward in terms of both clinical practice and scientific inquiry. Areas for future research include phenomenology, epidemiology, modulating factors, neurophysiological underpinnings, and treatment trials.

Keywords: misophonia, selective sound sensitivity syndrome, hyperacusis, neurodevelopmental disorders, Tourette syndrome, obsessive-compulsive spectrum

\section{What is misophonia?}

Shortly after the turn of the new millennium, Jastreboff et al first reported original clinical observations of subjects complaining of decreased sound tolerance with or without tinnitus. ${ }^{1-3}$ Interestingly, classic descriptions of hyperacusis (as characterized in patients who respond consistently to sounds above a certain intensity and whose reactions can be correlated with the physical parameters of the sound) did not fit majority of the reported cases. The authors therefore proposed misophonia as a new medical entity and defined misophonia as present when an abnormally strong reaction occurs to a sound with a specific pattern and/or meaning to an individual, with the context in which sound is presented frequently playing a role as well. Misophonic reactions often include autonomic arousal and unpleasant emotional experiences, such as anxiety, in response to specific sounds. Since the first scientific descriptions by Jastreboff et al, selective sound sensitivity syndrome has sometimes been used as a synonym of misophonia, alongside a range of layman's terms such as sound-rage, whereas decreased sound tolerance encompasses both misophonia and hyperacusis. ${ }^{4-6}$ Individuals with misophonia are sensitive to a specific set of trigger sounds, which are usually recognized since childhood; these sounds tend to be trivial noises produced by other people, including gum popping, food chewing or crunching, nose sniffing, breathing, pen clicking, clock ticking, whistling, lip smacking, and finger or foot tapping. However, it is important to note that trigger sounds do not necessarily need to be produced by other people: the noise produced by a train or an airplane, distant sounds of engines, as well as sounds made by animals can all potentially result in misophonic 
reactions in selected individuals. Interestingly, in addition to auditory cues, the observation of specific movements (eg, fingers pointing, legs swinging, and hair twirling) can also trigger intense aversive responses associated with feelings of distress, disgust, irritability, and anger. ${ }^{7-10}$ In contrast to phonophobia, a specific case of misophonia in which fear is the dominant emotion experienced in response to sound, misophonia is characterized by feelings of anxiety, distress, and occasionally anger. A range of physical manifestations have been described, which can accompany these psychological reactions, including tightness or pain in the chest, arms, head, or entire body, increased muscular tone, diaphoresis, dyspnea, tachycardia, hypertension, and hyperthermia. ${ }^{6}$ In general, there is a perception that the media have occasionally popularized misophonia by propagating an idea of extreme reaction of young people to sound related to eating; although such cases exist, misophonia can indeed emerge in elderly people and to a vast variety of sound as well. Moreover, contrary to the media reports, misophonic reactions in the majority of cases are mild-to-moderate, and external reactions can typically be kept under control. Finally, although persons with misophonia have a relatively preserved insight into the disproportionate nature of their feelings and reactions to sounds/movements that the vast majority of people would consider irrelevant, misophonic symptoms can lead to significant impairment across school, work, family, and social domains. In fact, stimulus-avoidance and other dysfunctional behaviors triggered by the auditory cues often affect the person's ability to achieve daily life goals and enjoy interpersonal interactions. ${ }^{7-9}$

\section{The clinical spectrum of misophonia}

The majority of studies on misophonia to date have been case reports/series, and although more systematic studies have recently been conducted, potentially more informative additional population studies are still needed..$^{7-9,11}$ A large sample of 42 persons with misophonia was clinically characterized by Schröder et al in 2013. ${ }^{8}$ These authors developed the Amsterdam Misophonia Scale, a six-item self-report rating scale to assess the type and severity of misophonia symptoms. Although this instrument was developed based on the Yale-Brown Obsessive Compulsive Scale used for obsessivecompulsive disorder, it also maps onto a set of six criteria proposed for the diagnosis of misophonia: 1) the presence or anticipation of a specific sound, produced by a human being (eg, eating sounds, breathing sounds), provokes an impulsive aversive physical reaction, which starts with irritation or disgust that instantaneously becomes anger; 2) this anger initiates a profound sense of loss of self-control with rare but potentially aggressive outbursts; 3) the person recognizes that the anger or disgust is excessive, unreasonable, or out of proportion to the circumstances or the provoking stressor; 4) the person tends to avoid the misophonic situation, or if he/she does not avoid it, endures encounters with the misophonic sound situation with intense discomfort, anger, or disgust; 5) the person's anger, disgust, or avoidance causes significant distress (ie, it bothers the person for whom he or she has the anger or disgust) or significant interference in the person's day-to-day life (eg, the anger or disgust may make it difficult for the person to perform important tasks at work, meet new friends, attend classes, or interact with others); and 6) the person's anger, disgust, and avoidance are not better explained by another disorder, such as obsessive-compulsive disorder (eg, disgust in someone with an obsession about contamination) or posttraumatic stress disorder (eg, avoidance of stimuli associated with a trauma related to threatened death, serious injury, or threat to the physical integrity of self or others). ${ }^{8}$ According to the Diagnostic and Statistical Manual of Mental Disorders (DSM) criteria, rates of psychiatric conditions in this misophonia sample were as follows: mood disorder $7.1 \%$, Tourette syndrome $4.8 \%$, attention-deficit and hyperactivity disorder $4.8 \%$, trichotillomania $4.8 \%$, skin picking $2.4 \%$, panic disorder $2.4 \%$, and hypochondria $2.4 \%$. Although DSM axis-1 obsessive-compulsive disorder was diagnosed in $2.4 \%$ of subjects, more than half of the sample met diagnostic criteria for axis-2 obsessive-compulsive personality disorder (52.4\%). Based on their observations, Schröder et al suggested that misophonia is a primary nosological entity, which can be appropriately categorized within obsessive-compulsive spectrum disorders, a group of conditions sharing elements of obsessionality, compulsivity, and impulsivity. ${ }^{8,12,13}$ This definition of misophonia contrasts with the one originally proposed by Jastreboff et al according to which the six criteria developed by Schröder would capture only a subset of individuals with misophonia (those with obsessive-compulsive and impulsive features) rather than all cases. ${ }^{1-3}$ In fact, the work by Jastreboff et al provides evidence of exceptions to each one of the six criteria: the misophonic trigger can sometimes be produced by animals or machines instead of human beings, and reactive anger is not observed in all subjects; beyond anger, negative reactions such as irritation and annoyance can create tension, but many subjects are able to control the external manifestation of misophonic reaction and keep self-control (with extra effort), as aggressive outbursts are observed only in a subgroup of patients (usually of young age); although the anger or disgust 
is recognized as excessive, unreasonable, or out of proportion to the circumstances/provoking stressor in the majority of cases, some subjects believe that other people behave in an unreasonable/disrespectful manner toward them, and therefore, their feelings/reactions are normal and justified; misophonic reactions can frequently be limited to annoyance or mild discomfort, rather than intense discomfort, disgust, or occasionally anger, and sometimes individuals with misophonia decide not to participate in a given activity due to concerns that their behavior would be distressful to their loved ones; negative reactions can interfere with daily life, although they do not necessarily involve anger or disgust; the presence of psychiatric or psychological disorders (diagnosed in $\sim 5 \%$ of cases in a recent large study on 184 consecutive subjects with misophonia) does not seem to play a role in determining misophonic reactions. ${ }^{11}$

Other observations, however, raise the possibility that at least in some cases, misophonia could be attributed to (or associated with) neuropsychiatric disorders, despite preliminary evidence supporting the suggestion that sensory over-responsivity can occur as a sole diagnosis. ${ }^{14,15}$ Intriguingly, misophonic symptoms and sensory over-responsivity have been recently documented in the context of pediatric obsessive-compulsive disorder, ${ }^{16-18}$ as well as a number of neurodevelopmental conditions, including attentiondeficit hyperactivity disorder, autistic spectrum disorder, and Fragile X syndrome. ${ }^{19-21}$

Furthermore, atypical sensory responses have been described in young patients with tic disorders, a group of conditions characterized by repetitive sudden, rapid, nonrhythmic movements (motor tics) or vocalizations (vocal of phonic tics). ${ }^{22,23}$ Tourette syndrome is a neurodevelopmental disorder characterized by chronic multiple motor and vocal/phonic tics and, in the majority of patients, specific obsessive-compulsive symptoms..$^{24,25}$ A recently published report from the International College of Obsessive-Compulsive Spectrum Disorders revealed that the most common comorbidity of obsessivecompulsive disorder in terms of obsessive-compulsive-related disorders was tic disorder (12.5\%), suggesting a bidirectional link between these patient populations where symptoms of misophonia have been reported. ${ }^{26}$ Importantly, although overt tics are the defining feature of Tourette syndrome, patients commonly report specific urges to tic, which are characterized by a strong sensory component. ${ }^{27,28}$ Premonitory urges are difficult to describe for many patients and result in unpleasant sensations, which increase in severity and are transiently relieved by tic expression. ${ }^{29-32} \mathrm{An}$ article published in 1994 by Michael Kane, a graduate student with Tourette syndrome, offered useful insights into these experiences, which can be perceived as more bothersome than the tics themselves and bear resemblance with misophonia-related discomfort (eg, urge to flee, comment on the noise, or otherwise avoid the noise). ${ }^{33}$ Based on his introspective case study, Kane proposed that the premonitory urges that precede tics are manifestations of sensory hyperawareness. In his words,

Perhaps the best description for the sensory state of Tourette syndrome is a somatic hyperattention: it is not as itch-like as it is an enduring somatosensory bombardment. ${ }^{33}$

Although a recent publication showed that subjects with misophonia can have enhanced autonomic reactivity to a sound, but not to other sensory stimuli, ${ }^{7}$ the subjective experiences described by these subjects following exposure to trigger sounds share qualitative features with the sensory symptoms reported by patients with tic disorders. Moreover, patients with Tourette syndrome often report the phenomenon of site sensitization, in which they become acutely aware of, distracted, and distressed by faint sensory stimuli, which include the auditory modality. ${ }^{34}$ A study on 28 patients with Tourette syndrome revealed that $70 \%$ reported heightened sensitivity to auditory, tactile, and/or visual stimuli, including noise from the television, electrical appliances, and loud settings such as parties. ${ }^{35}$ A more recent study using standardized questionnaires and in-depth interviews with 19 adult patients with Tourette syndrome and 19 aged-matched healthy controls showed that $80 \%$ of the patients described a heightened sensitivity to external stimuli in at least one sensory modality, namely smell (70\%), tactile perception (65\%), light (60\%), sound (55\%), and taste $(50 \%)$. Interestingly, these patients reported that stimuli that were faint, repetitive, or constant and nonsalient were the most bothersome, whereas intense stimuli were perceived as less troublesome ${ }^{36}$ Recent reports have also suggested that misophonic symptoms can be found in the context of two of the most common psychiatric comorbidities of Tourette syndrome, in addition to obsessive-compulsive disorder, generalized anxiety disorder, ${ }^{37,38}$ and schizotypal personality disorder. ${ }^{39,40}$ Overall, although there is preliminary evidence supporting the suggestion that misophonia can occur as a sole diagnosis, these observations raise the possibility that in some cases, it could also be attributed to underlying neuropsychiatric disorders.

\section{An elusive pathophysiology}

Research into the neurobiological underpinnings of misophonia is still in its infancy, and there are still a number of unanswered questions regarding the clinical characterization of this condition (Table 1). For example, the variability of 
Table I Suggestions for future research into misophonia

\begin{tabular}{ll}
\hline Domain & Research question \\
\hline Epidemiology & Prevalence in the general population \\
Phenomenology & Clinical characteristics of misophonic triggers \\
& Clinical characteristics of response to triggers \\
& Clinical correlates and comorbidities \\
& Factors modulating the misophonic response \\
& Psychological aspects \\
Henetics & Hereditary patterns \\
Pathophysiology & Neurophysiological processes \\
& Neuroanatomical correlates \\
& Structural and functional connectivity \\
Treatment & Neurochemical pathways involved \\
& Behavioral strategies \\
& Pharmacological options \\
& Other treatment modalities (biofeedback, etc) \\
\hline
\end{tabular}

the time course (onset, natural history) seems to depend on both life circumstances and degrees of exposure to trigger sounds. ${ }^{4,8}$ However, it is unclear which factors contribute to individual differences in severity of responses to misophonic triggers and in the nature of the triggers themselves. Both intrinsic and extrinsic factors, including family accommodation and exposure to visual stimuli typically associated with sound (eg, seeing somebody eating), ${ }^{11,15}$ seem to play a role in determining interindividual variability in the clinical presentation, which could be reflected in heterogeneous pathophysiological processes underlying misophonia. There is also uncertainty with regard to a possible genetic component to misophonia, as early observations identified the condition in multiple family members. ${ }^{4}$

Existing literature highlights broad similarities with tinnitus, a symptom that is often considered in the differential diagnosis of misophonia. ${ }^{1-3,6}$ According to Jastreboff and Hazell, both conditions could share a pattern of increased connectivity between auditory and limbic structures, resulting in heightened reactions to sounds. ${ }^{41}$ A comprehensive description of this interesting pathophysiological model, including proposed mechanisms by which conditioned reflexes can result in enhanced functional connections between the auditory system and other systems in the brain, particularly the limbic and autonomic nervous systems, has been included in recent publications by Jastreboff and Jastreboff. ${ }^{42,43}$ However, in misophonia, clinical manifestations are triggered by external, mostly human-produced sounds and situations, whereas persons who experience tinnitus report distress related to internally perceived, abstract sounds. Moreover, it is known that the majority of people can experience general and unelaborated emotional reactions to a range of annoying sounds: among the most familiar examples are the sounds of train wheels on rail tracks, fingernails on glass, or chalk on blackboard. ${ }^{44-46}$ It is possible that symptoms of misophonia reflect a more extreme subjective discomfort and physiological response to these and other emotionally evocative stimuli. This hypothesis would be consistent with the mentioned overlap with neurodevelopmental disorders, including Tourette syndrome, where sensory hyperawareness accompanied by subjective distress has been reported. The pathophysiology of the anomalous physiological/autonomic effects in the context of misophonia has recently been investigated: a study by Edelstein et al showed increased autonomic responses to auditory (but not visual) stimuli in six subjects with misophonia, compared to typically developed controls. ${ }^{7}$ Albeit in a small cohort, findings from this study using skin conductance responses provided an objective corroboration to subjective reports that specific sounds evoke intense emotional and physical reactions.

Evidence from the pharmacological modulation of the severity of misophonic symptoms might offer some insight on its pathophysiological mechanisms. Alcohol can alleviate the intensity of misophonia, whereas caffeine, a substance known to reduce auditory sensory gating in a paired-click auditory paradigm, ${ }^{47}$ has been reported to have opposite effects on symptom severity. ${ }^{7}$ Importantly, these notions are derived from early observations that are to be interpreted with caution until future studies on larger populations have been conducted.

\section{Treatment approaches}

Since the first documentation of misophonia cases in the scientific literature, there have been few treatment studies or empirical reports that could provide an evidence base for the treatment of its distressing symptoms. Until recently, ${ }^{11}$ there have been no large empirical treatment studies to evaluate the efficacy of therapeutic approaches to misophonia. Moreover, the lack of a coherent theoretical and etiological framework has hindered the identification of effective management strategies. Analysis of the coping strategies adopted by persons with misophonia have revealed that avoidance (removing self from distressing situations) and other socially dysfunctional behaviors (challenging the person who generates the trigger noise) can be replaced by more positive approaches. These include mimicry to cancel out the trigger sound, use of earplugs, headsets, or music, focusing consciousness to own sounds, self-distraction, and positive internal dialogs. ${ }^{7}$ Jastreboff and Jastreboff recently reported a success rate of $83 \%$ in a large treatment study of 184 patients recruited at a 
specialist center. ${ }^{11}$ The treatment approach followed general tinnitus retraining therapy principles, with additional use of four specific protocols for misophonia, sometimes including multisensory stimulation, and was based on active extinction of conditioned reflexes with incorporating principle of complex conditioned stimuli. ${ }^{11,48}$

Coming from a different perspective and building on the phenomenological similarity between misophonia and obsessive-compulsive spectrum disorders, Webber and Storch recently proposed a theoretical model based on the central role of anxiety and distress in order to explore potentially helpful treatment interventions. ${ }^{10}$ According to this model, the auditory or visual clues act as triggers for a negative emotional reaction (involving distress, anger, or anxiety) that is negatively reinforced by the behavioral response. The few published treatment studies for misophonia have limitations in scope, methodology, and sample size. The therapeutic approaches used in these studies ranged from psychoeducation and habituation to exposure and response prevention. ${ }^{49}$ The latter could be more effective in patients who experience significant anxiety and distress in response to the misophonic triggers, whereas patients who report severe anger or rage may be more likely to benefit from cognitive restructuring or stress inoculation. ${ }^{50}$ Other possibilities to explore in the treatment of misophonia are mindfulness- and acceptance-based approaches, such as dialectical behavior therapy and acceptance and commitment therapy. ${ }^{51}$ Overall, it seems likely that treatment plans need to be tailored to the needs of individual patients. It is also possible that persons who report misophonia in the context of neurodevelopmental and/or anxiety disorders could respond to pharmacological agents modulating serotonergic and/or antidopaminergic pathways, although these speculative suggestions need to be tested in clinical trials. ${ }^{4}$

\section{Physiological state, symptom, or disorder?}

The reviewed literature suggests a number of open questions about the exact nature of misophonia. According to the definition originally proposed by Jastreboff, misophonia is not to be considered a pathology or a psychological/psychiatric problem. ${ }^{1-3}$ Therefore, misophonic reactions could be induced in any person by creating an association between specific patterns and any type of sounds with negative reinforcement: it is enough that certain sounds regularly accompany situations of emotional stress, when the subject experiences pain or other negative emotions (for instance, as a result of hyperacusis or tensor tympanic syndrome, annoyance, anxiety) or beliefs (such as the belief that a specific sound will enhance tinnitus, produce hearing loss, or is produced by a person who is perceived in a negative manner). Real-life examples include the sound of the steps of the stepmother who is following the subject and makes his/her life miserable; the sound of kissing made by a sibling and accompanied by negative comments to irritate the subject; the sound of a train passing by after the subject has been in a car accident where the car was hit by a train; the clicking sound made by the claws of a cat walking over hard surfaces near the subject, who dislikes cats jumping on the table, etc. ${ }^{42}$ This definition challenges the subsequently proposed views that misophonia is a discrete/idiopathic condition (which can present with comorbid psychiatric disorders $)^{8}$ or a symptomatic manifestation of an underlying psychiatric disorder, at least in a proportion of cases. ${ }^{4}$ If confirmed by future systematic studies in large populations, the presence of high rates of comorbidity would go against the argument that misophonia should be labeled as a primary diagnosis. In fact, it would suggest that it is a symptom manifestation of other underlying or comorbid diagnoses and should more appropriately be labeled as a symptom, rather than as a stand-alone diagnosis. Either way, the addition of misophonia to nosographic classification systems of psychiatric disorders, such as the DSM, would require careful consideration. This issue was raised in a poignant way by Ronald Pies in a relevant commentary on the appropriateness of designating Internet addiction as a mental disorder in the fifth edition of the DSM (DSM-V). Pies eloquently argued that in recent years psychiatric nosography proliferated in an unprecedented and apparently unstoppable way, leading the general public to believe that the definitions of psychiatric disorders are settled by "vote by committee" in the academic equivalent of the "smoke-filled room". ${ }^{52}$ Needless to say, such a view, albeit possibly exaggerated, can affect public trust in psychiatry to a significant extent.

Although misophonia is believed to be far from rare, 8,42 this topic has attracted relatively little academic interest to date. Encouraging trends include the First International Conference on Hyperacusis, which was organized by the tinnitus and hyperacusis therapy team of the Royal Surrey County Hospital and held at Birkbeck College (University of London) in 2013..$^{53}$ This landmark event was followed by a highly successful second scientific meeting held at the same premises in 2015. There is general awareness that further research needs to be conducted before firm conclusions can be drawn with regard to both the pathophysiology and treatment of misophonia. Ideally, a comprehensive research agenda should also encompass epidemiological, phenomenological, and genetic 
aspects (Table 1). It is hoped that by combining qualitative and quantitative data from large populations, it will become possible to fully elucidate the hidden nature of this intrusive condition and alleviate the distress that it can cause.

\section{Acknowledgment}

Gratitude is expressed to the anonymous reviewers for their valuable comments.

\section{Disclosure}

The authors report no conflicts of interest in this work.

\section{References}

1. Jastreboff PJ. Tinnitus habituation therapy (THT) and tinnitus retraining therapy (TRT). In: Tyler RS, editor. Tinnitus Handbook. San Diego: Singular, Thomson Learning; 2000:357-376.

2. Jastreboff MM, Jastreboff PJ. Components of decreased sound tolerance: hyperacusis, misophonia, phonophobia. ITHS News Lett. 2001; 2:5-7.

3. Jastreboff PJ, Jastreboff MM. Tinnitus retraining therapy for patients with tinnitus and decreased sound tolerance. Otolaryngol Clin North Am. 2003;26:321-336.

4. Cavanna AE. What is misophonia and how can we treat it? Exp Rev Neurother. 2014;14:357-359.

5. Hadjipavlou G, Baer S, Lau A, Howard A. Selective sound intolerance and emotional distress: what every clinician should hear. Psychosom Med. 2008;70:739-740.

6. Schwartz P, Leyendecker J, Conlon M. Hyperacusis and misophonia: the lesser-known siblings of tinnitus. Minn Med. 2011;94:42-43.

7. Edelstein M, Brang D, Rouw R, Ramachandran VS. Misophonia: physiological investigations and case descriptions. Front Hum Neurosci. 2013;7:296.

8. Schröder A, Vulink N, Denys D. Misophonia: diagnostic criteria for a new psychiatric disorder. PLoS One. 2013;1:e54706.

9. Wu MS, Lewin AB, Murphy TK, Storch EA. Misophonia: incidence, phenomenology, and clinical correlates in an undergraduate student sample. J Clin Psychol. 2014;70:994-1007.

10. Webber TA, Storch EA. Toward a theoretical model of misophonia. Gen Hosp Psychiatry. 2015;37(4):369-370.

11. Jastreboff PJ, Jastreboff MM. Treatments for decreased sound tolerance (hyperacusis and misophonia). Semin Hear. 2014;35:105-120.

12. Hollander E, Wong CM. Obsessive-compulsive spectrum disorders. J Clin Psychiatry. 1995;56(suppl 4):3-6.

13. Starcevic V, Janca A. Obsessive-compulsive spectrum disorders: still in search of the concept-affirming boundaries. Curr Opin Psychiatry. 2011;24:55-60.

14. Reynolds S, Lane SJ. Diagnostic validity of sensory over-responsivity: a review of the literature and case reports. $J$ Autism Dev Disord. 2008;38:516-529.

15. Johnson PL, Webber TA, Wu MS, Lewin AB, Murphy TK, Storch EA. When selective audiovisual stimuli become unbearable: a case series on pediatric misophonia. Neuropsychiatry. 2013;3:569-575.

16. Hazen EP, Reichert EL, Piacentini JC, et al. Sensory intolerance as a primary symptom of pediatric OCD. Ann Clin Psychiatry. 2008;20: 199-203.

17. Lewin AB, Wu MS, Murphy TK, Storch EA. Sensory over-responsivity in pediatric obsessive compulsive disorder. J Psychopathol Behav Assess. 2015;37:134-143.

18. Webber TA, Johnson PL, Storch EA. Pediatric misophonia with comorbid obsessive-compulsive spectrum disorders. Gen Hosp Psychiatry. 2014;36:231.e1-e2.
19. Reynolds S, Lane SJ. Sensory overresponsivity and anxiety in children with ADHD. Am J Occup Ther. 2009;63:433-440.

20. Green SA, Ben-Sasson A. Anxiety disorders and sensory overresponsivity in children with autism spectrum disorders: is there a causal relationship? J Autism Dev Disord. 2010;40:1495-1504.

21. Tavassoli T, Miller LJ, Schoen SA, Nielsen DM, Baron-Cohen S. Sensory over-responsivity in adults with autism spectrum conditions. Autism. 2014;18:428-432.

22. Hazen EP, Reichert EL, Piacentini JC, et al. Case series: sensory intolerance as a primary symptom of pediatric OCD. Ann Clin Psychiatry. 2008;20:199-203.

23. Neal M, Cavanna AE. Selective sound sensitivity syndrome (misophonia) in a patient with Tourette syndrome. $J$ Neuropsychiatry Clin Neurosci. 2013;25(1):e1.

24. Cavanna AE, Rickards HE. The psychopathological spectrum of Gilles de la Tourette syndrome. Neurosci Biobehav Rev. 2013;37:1008-1015.

25. Eddy CM, Cavanna AE. Tourette syndrome and obsessive compulsive disorder: compulsivity along the continuum. J Obsess Compuls Rel Disord. 2014;3:363-371.

26. Lochner C, Fineberg NA, Zohar J, et al. Comorbidity in obsessivecompulsive disorder (OCD): a report from the International College of Obsessive-Compulsive Spectrum Disorders (ICOCS). Compr Psychiatry. 2014;55:1513-1519.

27. Rajagopal S, Seri S, Cavanna AE. Premonitory urges and sensorimotor processing in Tourette syndrome. Behav Neurol. 2013;27:65-73.

28. Tinaz S, Belluscio BA, Malone P, van der Veen JW, Hallett M, Horovitz SG. Role of the sensorimotor cortex in Tourette syndrome using multimodal imaging. Hum Brain Mapp. 2014;35:5834-5846.

29. Eddy CM, Cavanna AE. Premonitory urges in adults with complicated and uncomplicated Tourette syndrome. Behav Modif. 2013;38:264-275.

30. Crossley E, Cavanna AE. Sensory phenomena: clinical correlates and impact on quality of life in adult patients with Tourette syndrome. Psychiatry Res. 2013;209:705-710.

31. Crossley E, Seri S, Stern JS, Robertson MM, Cavanna AE. Premonitory urges for tics in adult patients with Tourette syndrome. Brain Dev. 2014;36:45-50.

32. Reese HE, Scahill L, Peterson AL, et al. The premonitory urge to tic: measurement, characteristics, and correlates in older adolescents and adults. Behav Ther. 2014;45:177-186.

33. Kane MJ. Premonitory urges as "attentional tics" in Tourette's syndrome. J Am Acad Child Adolesc Psychiatry. 1994;33:805-808.

34. Leckman JF, Bloch MH, Sukhodolsky DG, Scahill L, King RA. Phenomenology of tics and sensory urges: the self under siege. In: Martino D, Leckman JF, editors. Tourette Syndrome. Oxford: Oxford University Press; 2013:3-25.

35. Cohen AJ, Leckman JF. Sensory phenomena associated with Gilles de la Tourette's syndrome. J Clin Psychiatry. 1992;53:319-323.

36. Belluscio BA, Jin L, Watters V, Lee TH, Hallett M. Sensory sensitivity to external stimuli in Tourette syndrome patients. Mov Disord. 2011;26:2538-2543.

37. Khalifa N, Von Knorring A-L. Tourette syndrome and other tic disorders in a total population of children: clinical assessment and background. Acta Paediatr. 2005;94:1608-1614.

38. Conelea CA, Carter AC, Freeman JB. Sensory over-responsivity in a sample of children seeking treatment for anxiety. J Dev Behav Pediatr. 2014;35:510-521.

39. Cavanna AE, Robertson MM, Critchley HD. Schizotypal personality traits in Gilles de la Tourette syndrome. Acta Neurol Scand. 2007; 116:385-391.

40. Ferreira GM, Harrison BJ, Fontenelle LF. Hatred of sounds: misophonic disorder or just an underreported psychiatric symptom? Ann Clin Psychiatry. 2013;25:271-274.

41. Jastreboff PJ, Hazell J. Tinnitus Retraining Therapy: Implementing the Neurophysiological Model. New York: Cambridge University Press; 2004.

42. Jastreboff PJ, Jastreboff MM. Using TRT to treat hyperacusis, misophonia and phonophobia. ENT Audiol News. 2013;21:88-90. 
43. Jastreboff PJ, Jastreboff MM. Decreased sound tolerance: hyperacusis, misophonia, diplacousis, and polyacousis. Handb Clin Neurol. 2015; 129:375-387.

44. Halpern L, Blake R, Hillenbrand J. Psychoacoustics of a chilling sound. Percept Psychophys. 1986;39:77-80.

45. Zald DH, Pardo JV. The neural correlates of aversive auditory stimulation. Neuroimage. 2002;16:746-753.

46. Kumar S, von Kriegstein K, Friston K, Griffiths TD. Features versus feelings: dissociable representations of the acoustic features and valence of aversive sounds. J Neurosci. 2012;32:14184-14192.

47. Ghisolfi ES, Schuch A, Strimitzer IM Jr, et al. Caffeine modulates P50 auditory sensory gating in healthy subjects. Eur Neuropsychopharmacol. 2006;16(3):204-210.

48. Jastreboff MM, Jastreboff PJ. Decreased sound tolerance and tinnitus retraining therapy (TRT). Aust N Z J Audiol. 2002;24:74-84.
49. Bernstein RE, Angell KL, Dehle CM. A brief course of cognitive behavioural therapy for the treatment of misophonia: a case example. Cogn Behav Ther. 2013;6:e10-e13.

50. Beck R, Fernandez E. Cognitive-behavioral therapy in the treatment of anger: a metaanalysis. Cogn Ther Res. 1998;22:63-74.

51. Schneider RL, Arch JJ. Potential treatment targets for misophonia. Gen Hosp Psychiatry. 2015;37:370-371.

52. Pies R. Should DSM-V designate "internet addiction" a mental disorder? Psychiatry. 2009;6:31-37.

53. Aazh H, McFerran D, Salvi R, Prasher D, Jastreboff M, Jastreboff P Insights from the First International Conference on Hyperacusis: causes, evaluation, diagnosis and treatment. Noise Health. 2014;16:123-126.
Neuropsychiatric Disease and Treatment

\section{Publish your work in this journal}

Neuropsychiatric Disease and Treatment is an international, peerreviewed journal of clinical therapeutics and pharmacology focusing on concise rapid reporting of clinical or pre-clinical studies on a range of neuropsychiatric and neurological disorders. This journal is indexed on PubMed Central, the 'PsycINFO' database and CAS,

\section{Dovepress}

and is the official journal of The International Neuropsychiatric Association (INA). The manuscript management system is completely online and includes a very quick and fair peer-review system, which is all easy to use. Visit http://www.dovepress.com/testimonials.php to read real quotes from published authors.

Submit your manuscript here: http://www.dovepress.com/neuropsychiatric-disease-and-treatment-journal 